\title{
Exclusive production observed at the CMS experiment
}

\author{
Ruchi Chudasama (for the CMS Collaboration)* \\ Nuclear Physics Division \\ Bhabha Atomic Research Center \\ Mumbai, India \\ E-mail: ruchi.physics@gmail.com
}

Exclusive $\mathrm{W}^{+} \mathrm{W}^{-}$pair production in photon-photon collisions during the pp runs at 7 and $8 \mathrm{TeV}$ are observed and used to put constraints on the Anomalous Quartic Gauge Couplings. During the proton lead collisions in photon-induced vector meson production is observed via the decay of upsilon into two muons. The slope of the squared $\mathrm{p}_{\mathrm{T}}$ distribution is measured to determine the size of the production region.

XXIV International Workshop on Deep-Inelastic Scattering and Related Subjects

11-15 April, 2016

DESY Hamburg, Germany

${ }^{*}$ Speaker. 


\section{Introduction}

The CMS detector provides a very wide range of opportunities to study high-energy photoninduced interactions with proton and/or ion beams, due to the high energy and large integrated luminosities available at the LHC. Different exclusive particle production processes at high energies have been studied $[1,2,3]$. The very recent measurement of $\mathrm{W}$ pairs in the isolated electron-muon final-state have been used to extract the limit on anomalous quartic gauge coupling $[2,3]$. In addition, with the exclusive production of vector meson, nuclear gluon shadowing [4] and parton distribution function at very low $\mathrm{x}$ can be studied [9].

\section{Exclusive upsilon photoproduction in pPb collisions at $5.02 \mathrm{TeV}$}

Exclusive photoproduction of heavy vector mesons (Fig. 1,left) at very high photon-proton center-of-mass energies $\left(W_{\gamma p}\right)$ can be studied in ultraperipheral collisions (UPC) of protons (ions). Recently, CMS, ALICE [5] and LHCb [6] presented their measurements of exclusive heavy vector meson photoproduction at the LHC. Since the process occurs through $\gamma p$ or $\gamma P b$ interaction via the exchange of two-gluons with no net color transfer and thus, at leading order (LO), the cross section is proportional to the square of the gluon density in the target proton or ion. It provides a valuable probe of the gluon density at the small momentum fraction $x$ which is kinematically related to $W_{\gamma p}$ $\left(x=\left(M_{\Upsilon} / W_{\gamma p}\right)^{2}\right)$.
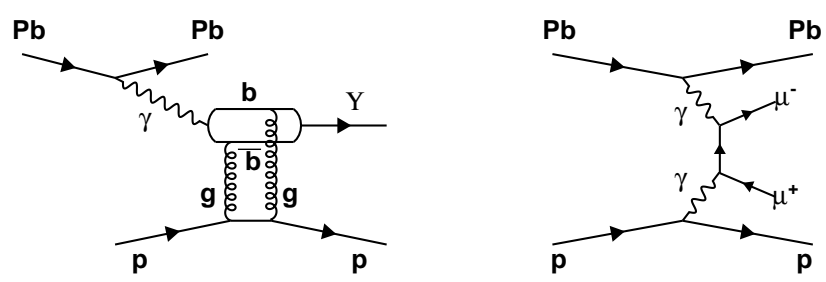

Figure 1: Diagrams representing exclusive $\Upsilon$ photoproduction (left), and exclusive dimuon QED continuum (right) in $\mathrm{pPb}$ collisions [9].

The exclusive photoproduction of $\Upsilon(1 \mathrm{~S}, 2 \mathrm{~S}, 3 \mathrm{~S})$ measurement has been measured in their dimuon decay channel in ultraperipheral collisions of protons and heavy ions (pPb) with the CMS experiment at $\sqrt{s_{\mathrm{NN}}}=5.02 \mathrm{TeV}$ for an integrated luminosity of $L_{\mathrm{int}}=33 \mathrm{nb}^{-1}$. The photoproduction cross section for $\Upsilon(n S)$ was measured as a function of $W_{\gamma p}$ in the range $91<W_{\gamma p}<826 \mathrm{GeV}$ which corresponds to the rapidity of the $\Upsilon$ meson in the range $|y|<2.2$ and $x$ values are of the order $x \sim 10^{-4}$ to $x \sim 1.3 \cdot 10^{-2}$. The dependence of the elastic $\Upsilon$ photoproduction cross section on the squared $\Upsilon$ transverse momentum approximating the four-momentum transfer at the proton vertex $\left(|t| \approx p_{\mathrm{T}}^{2}\right)$, can be parametrized with an exponential function $e^{-b|t|}$ at low values of $|t|$. The differential cross section $d \sigma / d t$, has been measured in the range $|t|<1.0(\mathrm{GeV} / \mathrm{c})^{2}$ and the b-slope parameter was estimated.

The UPC events were selected by applying dedicated HLT trigger which selects at least one muon in each event and at least one to six tracks. To select the exclusive $\Upsilon(\mathrm{nS})$ events offline, two muon tracks originating from the same primary vertex in each event were used. The muons 
were selected with $p_{\mathrm{T}}>3.3 \mathrm{GeV}$ and pseudorapidity $|\eta|<2.2$, in order to have high muon finding efficiency. The $p_{\mathrm{T}}$ of the muon pair was selected between 0.1 to $1 \mathrm{GeV}$. The lower cut on muon pair reduces the contamination from elastic QED background (Fig. 1,right) and higher cut on muon pair reduces the contamination from inelastic background (proton dissociation, inclusive $\Upsilon$, Drell-Yan). The rapidity of muon pair is restricted to $|y|<2.2$.

The dominant background contribution to exclusive $\Upsilon$ signal comes from QED, $\gamma \gamma \rightarrow \mu^{+} \mu^{-}$, which was estimated by STARLIGHT. The absolute prediction of QED was checked by comparing the data between invariant mass region $8-9.12$ and $10.64-12 \mathrm{GeV}$ for dimuon $p_{\mathrm{T}}<0.15 \mathrm{GeV}$ to the simulation. The contribution of non-exclusive background (inclusive $\Upsilon$, Drell-Yan and proton dissociation) was estimated by a data-driven method by selecting events with more than 2 tracks. This template was normalized to two muon track sample in the region of dimuon $p_{T}>1.5 \mathrm{GeV}$. Additional background in this analysis originates from a small contribution of exclusive $\gamma \mathrm{Pb} \rightarrow \Upsilon$ $\mathrm{Pb}$ events. The fraction of these events in the total number of exclusive $\Upsilon$ events was estimated using the reweighted STARLIGHT $\Upsilon$ MC sample. These backgrounds were subtracted from data to get the exclusive signal.

The background subtracted $|\mathrm{t}|$ and $\mathrm{y}$ distributions were used to measure the $\mathrm{b}$ parameter and estimate the exclusive $\Upsilon$ photoproduction cross-section as a function of $W_{\gamma p}$, respectively. The distributions were first unfolded to the region $0.01<|t|<1 \mathrm{GeV}^{2},|y|<2.2$, and muon $p_{\mathrm{T}}^{\mu}>$ 3.3 GeV, using the iterative Bayesian unfolding technique and it's further extraolated to transverse momenta zero by acceptance correction factor.

The differential $d \sigma / d t$ cross section is extracted for the combined three $\Upsilon(\mathrm{nS})$ states as shown in Fig. 2, according to

$$
\frac{\mathrm{d} \sigma_{\Upsilon}}{\mathrm{d}|t|}=\frac{N^{\Upsilon(\mathrm{nS})}}{\mathscr{L} \times \Delta|t|}
$$

where $|t|$ is approximated by the dimuon transverse momentum squared $p_{\mathrm{T}}^{2}, N^{\Upsilon(\mathrm{nS})}$ denotes the background-subtracted, unfolded and acceptance-corrected number of signal events in each $|t|$ bin, $\mathscr{L}$ is the integrated luminosity, and $\Delta|t|$ is the width of each $|t|$ bin. The cross section is fitted with an exponential function $N e^{-b|t|}$ in the region $0.01<|t|<1.0 \mathrm{GeV}^{2}$, using an unbinned $\chi^{2}$ minimization method. A value of $b=4.5 \pm 1.7$ (stat) \pm 0.6 (syst) $\mathrm{GeV}^{-2}$ is extracted from the fit. This result is in agreement with the value $b=4.3_{-1.3}^{+2.0}$ (stat) measured by the ZEUS experiment [13] for the photon-proton center-of-mass energy $60<W_{\gamma p}<220 \mathrm{GeV}$. The measured value of $b$ is also consistent with the predictions based on PQCD models [14].

The differential $\Upsilon(1 \mathrm{~S})$ photoproduction cross section $\mathrm{d} \sigma / \mathrm{d} y$ is extracted in four bins of dimuon rapidity according to

$$
\frac{d \sigma_{\Upsilon(1 \mathrm{~S})}}{d y}=\frac{f_{\Upsilon(1 \mathrm{~S})}}{\mathscr{B}\left(1+f_{\mathrm{FD}}\right)} \frac{N^{\Upsilon(\mathrm{nS})}}{\mathscr{L} \times \Delta y},
$$

where $N^{\Upsilon(\mathrm{nS})}$ denotes the background-subtracted, unfolded and acceptance-corrected number of signal events in each rapidity bin. The factor $f_{\Upsilon(1 \mathrm{~S})}$ describes the ratio of $\Upsilon(1 \mathrm{~S})$ to $\Upsilon(\mathrm{nS})$ events, $f_{\mathrm{FD}}$ is the feed-down contribution to the $\Upsilon(1 \mathrm{~S})$ events originating from the $\Upsilon(2 \mathrm{~S}) \rightarrow \Upsilon(1 \mathrm{~S})+X$ decays (where $X=\pi^{+} \pi^{-}$or $\left.\pi^{0} \pi^{0}\right), \mathscr{B}=(2.48 \pm 0.05) \%$ is the branching ratio for muonic $\Upsilon(1 S)$ decays, and $\Delta y$ is the width of the $y$ bin. 


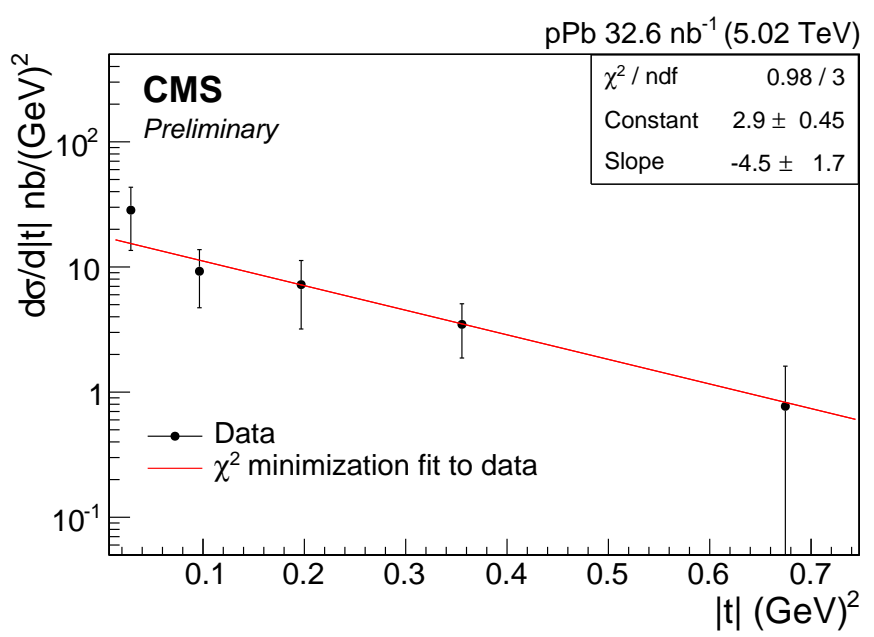

Figure 2: Differential $\Upsilon$ photoproduction cross section as a function of $|t|$ measured in $\mathrm{pPb}$ collisions at $\sqrt{s_{\mathrm{NN}}}=5.02 \mathrm{TeV}$ in the dimuon rapidity region $|y|<2.2$. The solid line represents the result of a fit with an exponential function $N e^{-b|t|}[9]$.

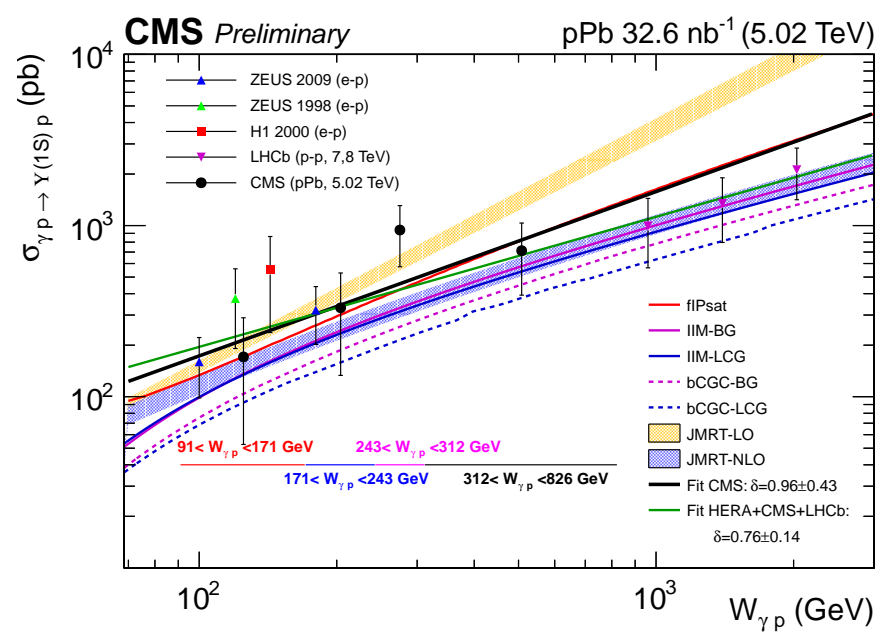

Figure 3: Cross section for exclusive $\Upsilon(1 S)$ photoproduction, $\gamma p \rightarrow \Upsilon(1 S) p$ as a function of photon-proton center-of-mass energy, $W_{\gamma p}[9]$.

The $f_{\Upsilon(1 \mathrm{~S})}$ fraction is used from the results of the inclusive $\Upsilon$ analysis [10]. The feed-down contribution of $\Upsilon(2 S)$ decaying to $\Upsilon(1 S)+\pi^{+} \pi^{-}$and $\Upsilon(1 S)+\pi^{0} \pi^{0}$ was estimated as $15 \%$ from the Starlight. The contribution from feed-down of exclusive $\chi_{b}$ states was neglected, as these double-pomeron processes are expected to be comparatively much suppressed in proton-nucleus collisions $[11,12]$.

The exclusive $\Upsilon(1 \mathrm{~S})$ photoproduction cross section as a function of $W_{\gamma p}$ as shown in Figure 3, 
is obtained by using,

$$
\sigma_{\gamma p \rightarrow \mathrm{r}(1 S) p}\left(W_{\gamma p}^{2}\right)=\frac{1}{\Phi} \frac{d \sigma_{\Upsilon(1 S)}}{d y}
$$

where $\Phi$ is the photon flux evaluated at the mean of the rapidity bin, estimated from STARLIGHT. The CMS data are plotted together with the previous measurements from H1 [7], ZEUS [8] and LHCb [2] data. It is also compared with different theoretical predictions of the JMRT model [14], factorized IPsat model $[15,16]$, IIM [17, 18] and bCGC model [19]. As $\sigma\left(W_{\gamma p}\right)$ is proportional to the square of the gluon PDF of the proton and the gluon distribution at low Bjorken $x$ is well described by a power law, the cross section will also follow a power law. Any deviation from such trend would indicate different behavior of gluon density function. We fit a power law $A \times(W / 400)^{\delta}$ with CMS data alone which gives $\delta=0.96 \pm 0.43$ and $A=655 \pm 196$ and is shown by the black solid line. The extracted $\delta$ value is comparable to the value $\delta=1.2 \pm 0.8$, obtained by ZEUS [4].

\section{Measurement of $\mathrm{W}^{+} \mathrm{W}^{-}$pair production in pp collisions at 7 and $8 \mathrm{TeV}$}

High energy photon interaction at LHC provide a unique opportunity to study exclusive production of $\mathrm{W}$ pairs. At leading order, quartic, t-channel and u-channel processes contributes to $\gamma \gamma \rightarrow W^{+} W^{-}$production (Fig. 4). The measurement of the quartic WW $\gamma \gamma$ coupling would provide an opportunity to look for any deviations from Standard Model (SM) and search for physics beyond SM. A genuine anomalous quartic gauge coupling (AQGC) is introduced via an effective lagrangian with two additional dimension- 6 terms containing the parameters $\mathrm{a}_{0}^{W}$ and $\mathrm{a}_{C}^{W}$. With the discovery of a light Higgs boson [22, 23, 24] a linear realization of the $\mathrm{SU}(2) \times \mathrm{U}(1)$ symmetry of the SM, spontaneously broken by the Higgs mechanism, is possible. Thus, the lowest order operators, where new physics may cause deviations in the quartic gauge boson couplings alone, are of dimension 8. By assuming the the anomalous WWZ $\gamma$ vertex vanishes, a direct relationship between the dimension- 8 and dimension- 6 couplings can be recovered [25]. In both dimension6 and dimension- 8 scenarios, the $\gamma \gamma \rightarrow W^{+} W^{-}$cross section increase quadratically with energy, therefore a dipole form factor is introduced to preserve unitarity.
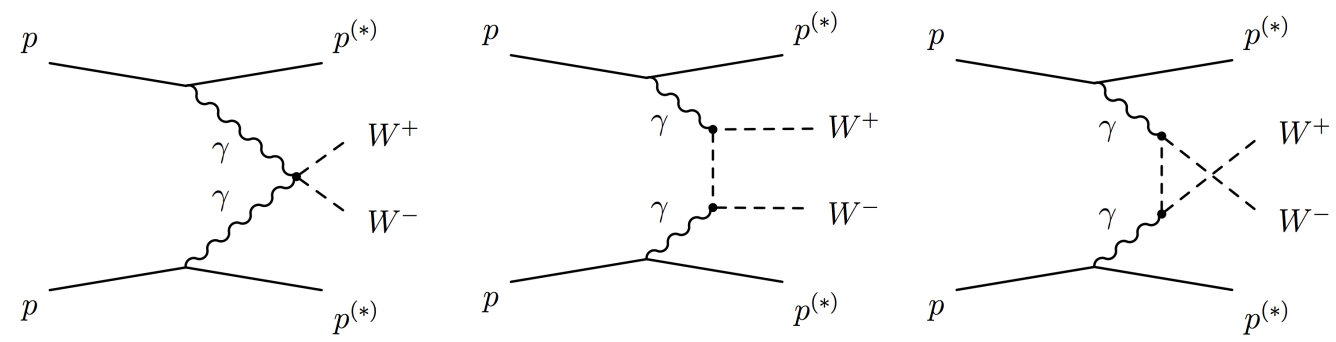

Figure 4: Quartic (left), t-channel (center), and u-channel (right) diagrams contributing to the $\gamma \gamma \rightarrow W^{+} W^{-}$ process at leading order in the SM [3].

CMS has carried out a measurement of exclusive and quasi-exclusive $\gamma \gamma \rightarrow W^{+} W^{-}$production, via $p p \rightarrow p^{(*)} W^{+} W^{-} p^{(*)} \rightarrow p^{(*)} \mu^{ \pm} e^{\mp}$ at 7 and $8 \mathrm{TeV}$, corresponding to luminosities of $5.5 \mathrm{fb}^{-1}$ and $19.7 \mathrm{fb}^{-1}$, respectively. The production of $\mathrm{W}$ pairs was measured in $\mu^{ \pm} e^{\mp}$ final 
state, since, $W^{+} W^{-} \rightarrow \mu^{+} \mu^{-}$or $W^{+} W^{-} \rightarrow e^{+} e^{-}$would be dominated by Drell-Yan events and $\gamma \gamma \rightarrow l^{+} l^{-}$production. The $\mu^{ \pm} e^{\mp}$ events were extracted by skimming the dataset with dedicated HLT trigger which selects two leptons with transverse momentum $p_{T}>17(8) \mathrm{GeV}$ for the leading (subleading) lepton. Offline, the events with the opposite-charge electron-muon pair originating from a common primary vertex that has no additional tracks associated with it were selected to remove the underlying event activity. The events with transverse momentum of the pair $\mathrm{p}_{T}\left(\mu^{ \pm} e^{\mp}\right)$ $>30 \mathrm{GeV}$ were selected to suppress backgrounds from $\tau^{+} \tau^{-}$production, including the exclusive and quasi-exclusive $\gamma \gamma \rightarrow \tau^{+} \tau^{-}$processes.

Simulations show that in high-mass $\gamma \gamma$ interactions one or both of the protons dissociate, which may result in events being rejected by the veto on extra tracks. To estimate this effect from data, a sample is selected where the dilepton invariant mass is greater than $160 \mathrm{GeV}$ so that $\mathrm{W}^{+} \mathrm{W}^{-}$pairs can be produced on shell. The ratio of the observed number of events to the calculated number of elastic $\mathrm{pp} \rightarrow \mathrm{p} l^{+} l^{-} \mathrm{p}$ events is used as a scale factor to calculate from the predicted elastic $\mathrm{pp} \rightarrow$ $\mathrm{pW}^{+} \mathrm{W}^{-} \mathrm{p}$ events the total number of $\mathrm{pp} \rightarrow \mathrm{p}^{*} \mathrm{~W}^{+} \mathrm{W}^{-} \mathrm{p}^{*}$ to be expected when including proton dissociation. The numerical value of the scale factor thus obtained is $\mathrm{F}=4.10 \pm 0.43$.
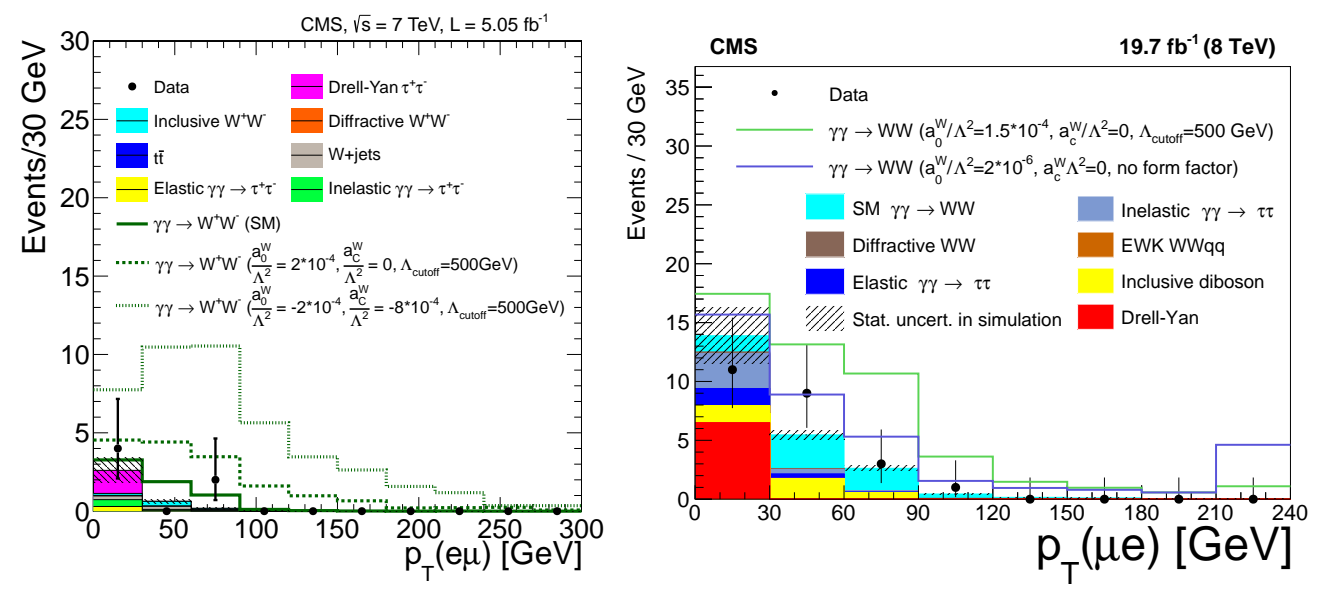

Figure 5: The $\mathrm{p}_{T}\left(\mu^{ \pm} e^{\mp}\right)$ distribution for events with zero extra tracks at $7 \mathrm{TeV}$ (left) [2] and at 8 TeV(right) [3].

Fig. 5 shows the $\mathrm{p}_{T}\left(\mu^{ \pm} e^{\mp}\right)$ distribution for events passing all other selection requirements. In the signal region with no additional tracks and $\mathrm{p}_{T}\left(\mu^{ \pm} e^{\mp}\right)>30 \mathrm{GeV}, 2$ events are observed at 7 $\mathrm{TeV}$ compared to the expectation of $2.2 \pm 0.4$ signal events and $0.84 \pm 0.15$ background events, corresponding to an observed (expected) significance of $0.8 \sigma(1.8 \sigma)$. While 13 events are observed at $8 \mathrm{TeV}$ compared to the expectation of $5.3 \pm 0.7$ signal events and $3.9 \pm 0.6$ background events, corresponds to a mean expected signal significance of $2.1 \sigma$. We combine the 7 and $8 \mathrm{TeV}$ results, treating all systematic uncertainties as fully uncorrelated between the two measurements, the resulting observed (expected) significance for the 7 and $8 \mathrm{TeV}$ combination is $3.4 \sigma(2.8 \sigma)$, constituting evidence for $\gamma \gamma \rightarrow W^{+} W^{-}$production in proton-proton collisions at the LHC. Interpreting the $8 \mathrm{TeV}$ results as a cross section multiplied by branching fraction to $\mu^{ \pm} e^{\mp}$ ) final states, corrected for all experimental efficiencies and extrapolated to the full space, yields :

$$
\sigma\left(\mathrm{pp} \rightarrow \mathrm{p}^{(*)} \mathrm{W}^{+} \mathrm{W}^{-} \mathrm{p}^{(*)} \rightarrow \mathrm{p}^{(*)} \mu^{ \pm} \mathrm{e}^{\mp} \mathrm{p}^{(*)}\right)=11.9_{-4.5}^{+5.6} \mathrm{fb}
$$


The corresponding 95\% confidence level (CL) upper limit obtained from the $7 \mathrm{TeV}$ data was $<10.6$ $\mathrm{fb}$, with a central value of $2.2_{-2.0}^{+3.3} \mathrm{fb}$.

The transverse momentum $\mathrm{p}_{T}\left(\mu^{ \pm} e^{\mp}\right)$ distribution was also used to search for sign of anomalous quartic gauge couplings. $\mathrm{p}_{T}\left(\mu^{ \pm} e^{\mp}\right)>100 \mathrm{GeV}$ is used at $7 \mathrm{TeV}$, while two bins, with boundaries $\mathrm{p}_{T}\left(\mu^{ \pm} e^{\mp}\right)=30-130 \mathrm{GeV}$ and $\mathrm{p}_{T}\left(\mu^{ \pm} e^{\mp}\right)>130 \mathrm{GeV}$, are used to set the limit on aQGC. Fig. 6 shows the excluded values of the anomalous coupling parameters $\mathrm{a}_{0}^{W} / \wedge^{2}$ and $\mathrm{a}_{C}^{W} / \wedge^{2}$ with $\wedge_{\text {cutoff }}=500 \mathrm{GeV}$. The exclusion regions are shown at $7 \mathrm{TeV}$ (outer contour), $8 \mathrm{TeV}$ (middle contour), and the $7+8 \mathrm{TeV}$ combination (innermost contour). The areas outside the solid contours are excluded by each measurement at $95 \%$ CL. The cross indicates the one-dimensional limits obtained for each parameter from the 7 and $8 \mathrm{TeV}$ combination, with the other parameter fixed to zero, more details can be found in [3].

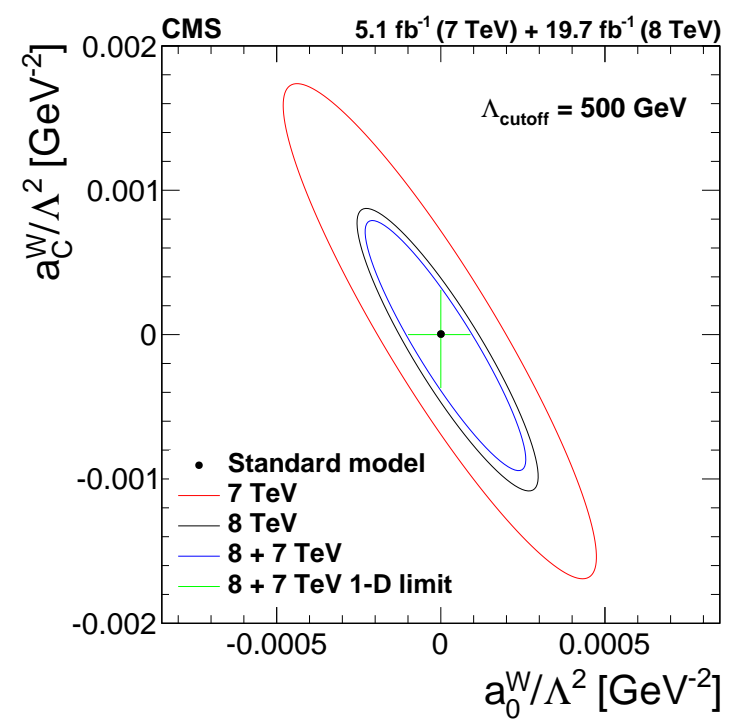

Figure 6: Excluded values of the anomalous coupling parameters $\mathrm{a}_{0}^{W} / \wedge^{2}$ and $\mathrm{a}_{C}^{W} / \wedge^{2}$ with $\wedge_{\text {cutoff }}=500$ $\mathrm{GeV}$. The areas outside the solid contours are excluded by each measurement at 95\% CL [3].

\section{Summary}

We reported the first measurement of the exclusive photoproduction of $\Upsilon(1 \mathrm{~S}, 2 \mathrm{~S}, 3 \mathrm{~S})$ mesons in the $\mu^{+} \mu^{-}$decay modes in ultraperipheral $\mathrm{pPb}$ collisions at $\sqrt{s_{\mathrm{NN}}}=5.02 \mathrm{TeV}$, corresponding to an integrated luminosity of $33 \mathrm{nb}^{-1}$. The exclusive photoproduction cross sections have been measured as a function of the photon-proton center of mass energy, bridging a previously unexplored region between HERA and LHCb measurements. Our data are compatible with a power law dependence of $\sigma\left(W_{\gamma p}\right)$, disfavouring faster rising predictions of LO pQCD. The spectral slope $\mathrm{b}$ has been extracted, in agreement with earlier measurements. Results have been also presented for exclusive and quasi-exclusive $\gamma \gamma \rightarrow W^{+} W^{-}$production in the $\mu^{ \pm} e^{\mp}$ final state in pp collisions at $\sqrt{s_{\mathrm{NN}}}=7$ and $8 \mathrm{TeV}$, corresponding to an integrated luminosity of $5.5 \mathrm{fb}^{-1}$ and $19.7 \mathrm{fb}^{-1}$, respectively. The observed yields and kinematic distributions are consistent with the SM prediction, with 
a combined significance over the background-only hypothesis of $3.4 \sigma$. No significant deviations from the SM are observed in the $\mathrm{p}_{T}\left(\mu^{ \pm} e^{\mp}\right)$ distribution.

\section{References}

[1] CMS Collaboration, JHEP 01 (2012) 052

[2] CMS Collaboration, JHEP 07 (2013) 116

[3] CMS Collaboration, arXiv: 1604.04464

[4] CMS Collaboration, arXiv: 1605.06966

[5] ALICE Collaboration, Phys. Rev. Lett. 113 (2014) 232504.

[6] LHCb Collaboration, JHEP 09 (2015) 084, arXiv:1505.08139.

[7] H1 Collaboration, Eur. Phys. B 46 (2006) 585.

[8] ZEUS Collaboration, Phys. Lett. B 680 (2009) 4.

[9] CMS Collaboration, FSQ-13-009

[10] CMS Collaboration, JHEP 04 (2014) 103.

[11] A. J. Schramm and D. H. Reeves, Phys. Rev. D 55 (1997) 7312.

[12] L. A. Harland-Lang, V. A. Khoze, M. G. Ryskin, and W. J. Stirling, Eur. Phys. J. C 69 (2010) 179.

[13] ZEUS Collaboration, Phys. Lett. B 708 (2012) 14.

[14] P. Jones, D. Martin, M. G. Ryskin, and T. Teubner, JHEP 11 (2013) 085.

[15] T. Lappi and H. Mantysaari, Phys. Rev. C 83 (2011) 065202.

[16] T. Lappi and H. Mantysaari, Phys. Rev. C 87.

[17] G. Sampaio dos Santos and M. V. T. Machado, Phys. Rev. C 89 (2014) 025201,(2013) 032201.

[18] G. Sampaio dos Santos and M. V. T. Machado, J. Phys. G42 (2015) 105001.

[19] V. P. Goncalves, B. D. Moreira, and F. S. Navarra, Phys. Lett. B 742 (2015) 172.

[20] O. J. P. Eboli, M. C. Gonzalez-Garcia and S. M. Lietti, Phys. Rev. D 69, 095005 (2004).

[21] O. J. P. Eboli, M. C. Gonzalez-Garcia and J. K. Mizukoshi, Phys. Rev. D 74, 073005 (2006)

[22] ATLAS Collaboration, Phys. Lett. B 716 (2012) 1

[23] CMS Collaboration, Phys. Lett. B 716 (2012) 30

[24] CMS Collaboration, JHEP 06 (2013) 081

[25] CMS Collaboration, Phys. Rev. D 90 (2014) 032008 